\title{
Group-based social skills interventions for adolescents with higher-functioning autism spectrum disorder: a review and looking to the future
}

\author{
This article was published in the following Dove Press journal: \\ Adolescent Health, Medicine and Therapeutics \\ 21 January 2013 \\ Number of times this article has been viewed
}

\author{
Camilla M McMahon' \\ Matthew D Lerner ${ }^{2,3}$ \\ Noah Britton ${ }^{4}$ \\ 'Department of Curriculum and \\ Instruction, Indiana University \\ Bloomington, Bloomington, \\ IN, USA; '2Department of Psychology, \\ University of Virginia, Charlottesville, \\ VA, USA; ${ }^{3}$ Department of Psychiatry \\ and Behavioral Neuroscience, \\ University of Chicago, Chicago, \\ IL, USA; ${ }^{4}$ Behavorial Sciences \\ Department, Bunker Hill Community \\ College, Charleston, MA, USA
}

\begin{abstract}
In this paper, we synthesize the current literature on group-based social skills interventions (GSSIs) for adolescents (ages 10-20 years) with higher-functioning autism spectrum disorder and identify key concepts that should be addressed in future research on GSSIs. We consider the research participants, the intervention, the assessment of the intervention, and the research methodology and results to be integral and interconnected components of the GSSI literature, and we review each of these components respectively. Participant characteristics (eg, age, IQ, sex) and intervention characteristics (eg, targeted social skills, teaching strategies, duration and intensity) vary considerably across GSSIs; future research should evaluate whether participant and intervention characteristics mediate/moderate intervention efficacy. Multiple assessments (eg, parent-report, child-report, social cognitive assessments) are used to evaluate the efficacy of GSSIs; future research should be aware of the limitations of current measurement approaches and employ more accurate, sensitive, and comprehensive measurement approaches. Results of GSSIs are largely inconclusive, with few consistent findings across studies (eg, high parent and child satisfaction with the intervention); future research should employ more rigorous methodological standards for evaluating efficacy. A better understanding of these components in the current GSSI literature and a more sophisticated and rigorous analysis of these components in future research will lend clarity to key questions regarding the efficacy of GSSIs for individuals with autism spectrum disorder.
\end{abstract}

Keywords: intervention, social skills, autism, group, review, methodology

\section{Introduction}

Autism spectrum disorder (ASD) is a developmental disability characterized by qualitative impairments in social interaction and communication, as well as restricted, repetitive behaviors, activities, and/or interests. ${ }^{1}$ Given the high prevalence of ASD diagnosis (one in 88 children), ASD has been recognized as an area of important public health concern, ${ }^{2}$ and the development of safe and effective interventions for individuals with ASD has been identified as a funding priority. ${ }^{3}$

While multiple intervention strategies have been used to promote social skills in adolescents with ASD, ${ }^{4}$ the present paper focuses on group-based social skills interventions (GSSIs). Broadly defined, a GSSI is an intervention that aims to improve the social skills of intervention participants and occurs in a group setting with many higher-functioning individuals with ASD (see Participants section) and at least one therapist or teacher. In this paper, we synthesize the literature on GSSIs for adolescents
Correspondence: Camilla M McMahon Department of Curriculum and Instruction, Indiana University Bloomington, 20I North Rose Avenue, Bloomington, IN 47405, USA

Email cammcmah@indiana.edu 
with higher-functioning ASD and identify key concepts that should be addressed in future research on GSSIs. We consider the research participants, the intervention, the assessment of the intervention, and the research methodology and results to be integral and interconnected components of the GSSI literature, and we discuss each of these components respectively.

This paper represents a selective review of the GSSI literature, based on a systematic search of the PsycINFO database. The terms "autism or autistic or pervasive developmental or Asperger," "intervention or training or treatment," and "social" were used as subject headings for the search, and the search was further restricted to peer-reviewed articles, articles written in the English language, articles published after 1990 (when the vast majority of the research in this literature has taken place), and articles focused on the schoolage, adolescent, and young-adult age-groups (who are most likely to receive GSSI interventions in real-world settings and who comprise the target population of adolescents). This search yielded 376 results.

Six criteria were used to determine which studies would be included in the current review: (1) the study specifically focused on individuals with ASD; (2) the study assessed the efficacy of an intervention (ie, reviews, meta-analyses, longitudinal follow-ups, theoretical papers, etc, were not included); (3) the intervention focused on social skill improvement (ie, interventions focused on challenging behaviors, comorbid symptoms, communication, etc, were not included); (4) the intervention was delivered in a group-based format, and groups were primarily composed of other individuals with ASD; (5) the intervention included at least some participants within the 10- to 20-year-old age range; and (6) the intervention data were not primarily analyzed using a single-subject design. A few studies identified by the PsycINFO search could not be located by the authors, and these studies are not represented in the current review. In addition, the authors added relevant studies to this review that were not captured through the PsycINFO search, such as manuscripts in press and others based on the authors' expertise in this field. See Table 1 for a summary of studies included in the literature review.

\section{Group-based social skills interventions: a review \\ Participants}

While some studies within the GSSI literature employ fewer than ten participants ${ }^{5-8}$ or more than 50 participants, ${ }^{9-12}$ the majority of GSSIs are evaluated using a total sample size of
10-50 participants. ${ }^{13-44}$ Almost all GSSIs employ a series of inclusion/exclusion criteria to ensure that participants are appropriate for the intervention. In addition to meeting diagnostic criteria, participants are most commonly required to have sufficient verbal language and/or have an IQ above a given threshold, 7,9,10,12,13,16,17,19,22,24-27,29,31-33,35,37-42,44 meet age or grade-level requirements, ${ }^{10,16,17,20-22,24-27,31,35,36,38,39,42-44}$ and show an absence of severe behavioral problems or comorbid psychopathology. ${ }^{10,17,19,21,22,24-28,31,32,35,41,43}$ While the previously mentioned inclusion/exclusion criteria are the most common, such criteria may also require that participants and/or their families are interested in or committed to participation in the intervention, $9,17,20,26,27,34,40,42$ participants spend at least some time in a general education setting, ${ }^{6,16,35,38,39,42}$ and caregivers report social skill deficits in the participants. $9,17,26,27,42$ Although rarely used as an inclusion/exclusion criterion, ${ }^{15,25}$ the majority of intervention participants are male, as ASD is more common in males than females. ${ }^{2}$ Overall, these inclusion/exclusion criteria are used to ensure that intervention participants are higher-functioning adolescents with ASD who are appropriate for a group-based intervention.

\section{Interventions}

Several key dimensions vary across GSSIs, including the social skills that are targeted by the intervention, the teaching strategies that are employed in the intervention, the duration and intensity of the intervention, the setting of the intervention, and the degree to which the intervention adheres to a manual or curriculum. While GSSIs may target a specific subset of social skills, they generally target social skills within the domains of nonverbal communication (eg, eye contact; facial expressions; posture; gestures), verbal communication (eg, tone of voice; humor and jokes; nonliteral language such as metaphors, sarcasm, and figures of speech), social interaction (eg, friendship; joining, maintaining, or leaving a social interaction; conversation; empathy), and/or social problem solving (eg, conflict in relationships; bullying and teasing; controlling negative emotions; good sportsmanship). ${ }^{5-44}$

The most widely used teaching strategy in GSSIs is the structured-learning approach. In this approach, social skills are taught via a didactic lesson and then modeled by a therapist or teacher. Intervention participants are given the opportunity to practice the skills via role-play and then receive feedback on their execution of the skills. The Skillstreaming Program ${ }^{12,30,32,33}$ and the Program for the Education and Enrichment of Relational Skills (PEERS) ${ }^{17,26,27}$ are two relatively well-known GSSIs that employ a structured-learning approach, among other GSSIs. ${ }^{5,7-10,15,16,19,23-25,34-40,42,44}$ Another 
teaching strategy that is gaining traction in the GSSI literature is social performance training. In social performance training, social skills are implicitly taught as intervention participants engage in targeted activity- or drama-based games; social skills are not explicitly taught via didactic instruction. ${ }^{29-31}$ Similarly, child-directed learning also favors implicit social skills instruction; this teaching strategy encourages intervention participants to collaboratively engage in an activity of interest, such as Lego play, that promotes regular, positive social interaction among group members. ${ }^{11,28,35} \mathrm{~A}$ final teaching strategy is that of social skills support group, in which participants discuss and reflect on their own social experiences and the social experiences of other participants. ${ }^{20,21,43}$

Regardless of the overall teaching strategy adopted by a GSSI, GSSIs may incorporate additional strategies to promote the maintenance of social skills after the intervention has ended and the generalization of social skills beyond the intervention setting. Often, GSSIs may have a parent intervention group that meets concurrently to the child intervention group or occasionally throughout the intervention; the goal of the parent intervention group may be to provide support and/or psychoeducational training for implementing the intervention curriculum at home. ${ }^{9,10,12,13,17-20,26,27,33,37,38,43}$ GSSIs may also include typically developing peers and/or siblings in the child intervention group; typically developing children may serve as a social role model for intervention participants, provide feedback on inappropriate social behaviors, and promote generalization of social skills to nonclinical settings. ${ }^{11,15,24,28,44}$ (Note that the current review focuses on GSSIs in which groups are primarily composed of individuals with ASD; however, there is a related body of literature that emphasizes peer-mediated social skill interventions, some of which also take place in a group context.) Finally, some GSSIs include structural elements within the curriculum to promote maintenance and generalization of social skills, such as setting up get-togethers with peers, ${ }^{17,26,27}$ engaging in intervention assignments at home, ${ }^{8,9,12,15,17,19,25-27,32,33,37,44}$ having monthly reunions for intervention participants, ${ }^{21}$ and participating in community outings during the intervention. $5,7,10,14,34$

The majority of GSSIs are implemented for 1-2 hours/ week..$^{7-11,13-22,24-28,30,34-40,42,44}$ These interventions are generally conducted during the academic year and may last for a few weeks $^{30}$ or span the academic year. ${ }^{16}$ A minority of GSSIs employ longer-lasting intervention sessions that tend to span fewer weeks, often during the summer months. These interventions typically last 5-6 hours/weekday over the course of 1 or 2 months..$^{12,29,31-33}$ In addition, almost all published interventions occur in clinic or university settings, ${ }^{5-28,30,32-41,43,44}$ the GSSI literature rarely includes interventions implemented in school or community settings. $29,31,42$

Finally, the majority of GSSIs are not manualbased and do not assess fidelity to the social skills curriculum. $^{5-9,11,14-16,18,20-23,28,34,36-41,43}$ For these interventions, it is not known whether therapists/teachers adhere to the social skills curriculum, such that all intervention participants receive the intended curriculum. A minority of GSSIs do assess fidelity to the intervention curriculum. ${ }^{10,12,13,17,24-27,30-33,44}$

\section{Assessments}

In the current literature, there are six categories of assessments that are regularly used to gauge the efficacy of a GSSI. Parent-report questionnaires $5,7,9,10,12-20,22-27,30-44$ tend to be quick and easy to administer, hence almost all GSSIs employ this assessment method. Child-report questionnaires $5,6,10,13,14,17,20-23,26,27,29,31,33-37,40,42,43$ and social cognitive assessments (ie, assessments that measure one's ability to process social information) $)^{5,7,8,12,13,15,17,18,25-27,31,33,37-39,42}$ are fairly straightforward to administer in a lab setting, and the majority of GSSIs employ one or both of these assessment methods. Teacher-report questionnaires, ${ }^{7,15,16,19,26,27,39,41,44}$ clinicianor intervention staff-report questionnaires, ${ }^{11,12,19,24,28,30,32,33}$ and observations of participants' behavior ${ }^{8,19,20,23,25,28,30,35,42}$ tend to be more logistically complicated assessment methods and are less frequently employed in the GSSI literature.

The specific measures that are used to indicate the efficacy of a GSSI are important to consider, as the reported efficacy of an intervention is dependent on the quality and suitability of its assessments. The Social Responsiveness Scale ${ }^{10,17,25,26,30,31,33,38-40,44,45}$ and Social Skills Rating System $^{7,9,15,17,26,27,30,31,42,46}$ are the most common adult-report questionnaires, along with informal questionnaires to indicate satisfaction with the intervention. $5,9,12,14,15,20,22-24,30,31,33-36$, 40,42-44 Other adult-report questionnaires that are sometimes used in this literature include the Behavior Assessment System for Children, ${ }^{12,32,33,47}$ Vineland Adaptive Behavior Scales, ${ }^{11,18,25,35,41,48}$ and various questionnaires designed by intervention staff that are specific to the intervention curriculum. ${ }^{10,12,16,17,26,27,33}$

Currently, there are no child-report questionnaires that are regularly used in the GSSI literature, although children are frequently asked to indicate their satisfaction with the intervention via informal questionnaires. 5,6,14,20,22,23,33,35,36,40,42,43 Child-report questionnaires that are occasionally used in the GSSI literature include the Beck Depression Inventory, ${ }^{21,31,49,50}$ Index of Peer Relations, ${ }^{20-22,51}$ State Trait Anxiety Inventory, ${ }^{21,22,52}$ 


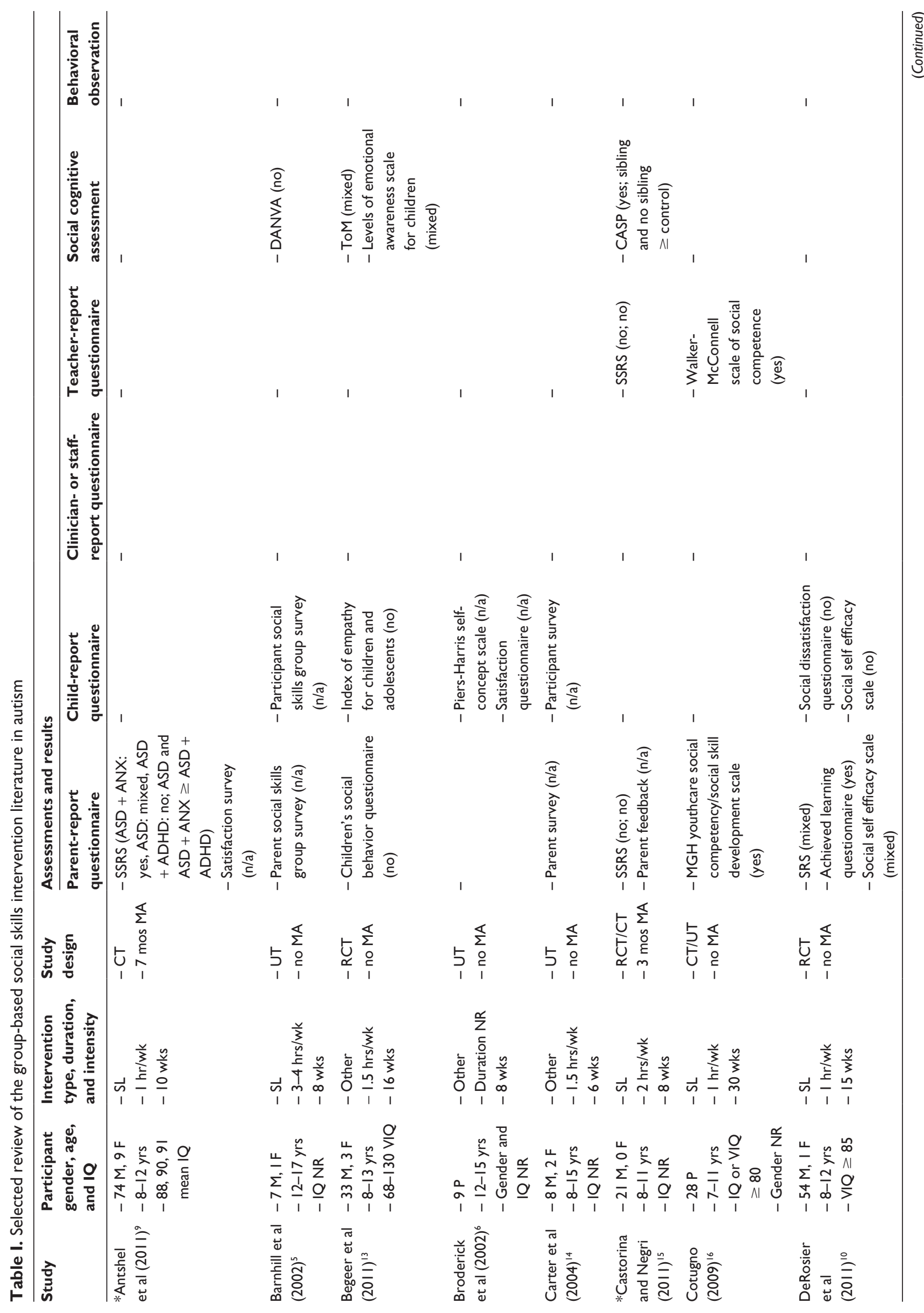




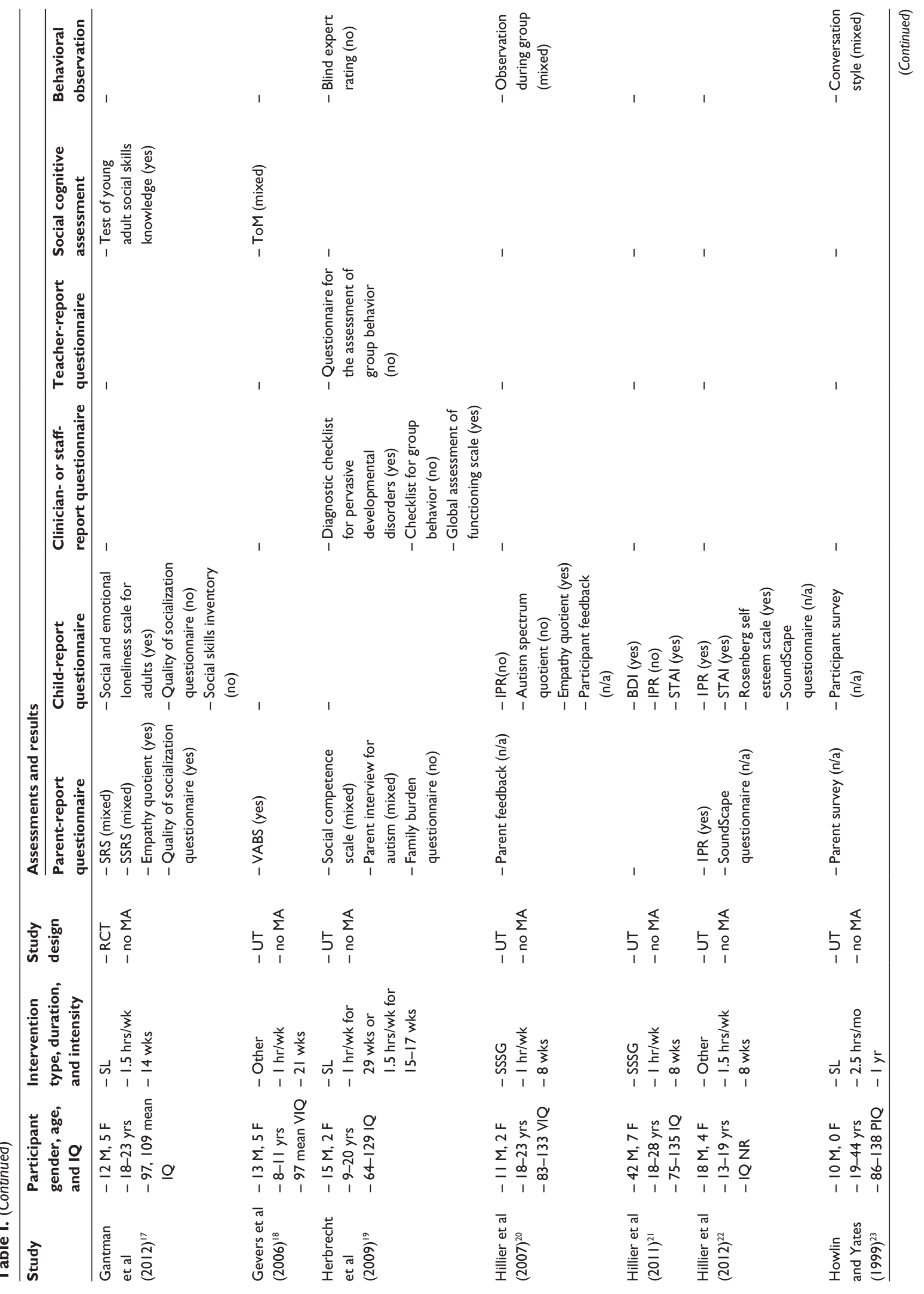



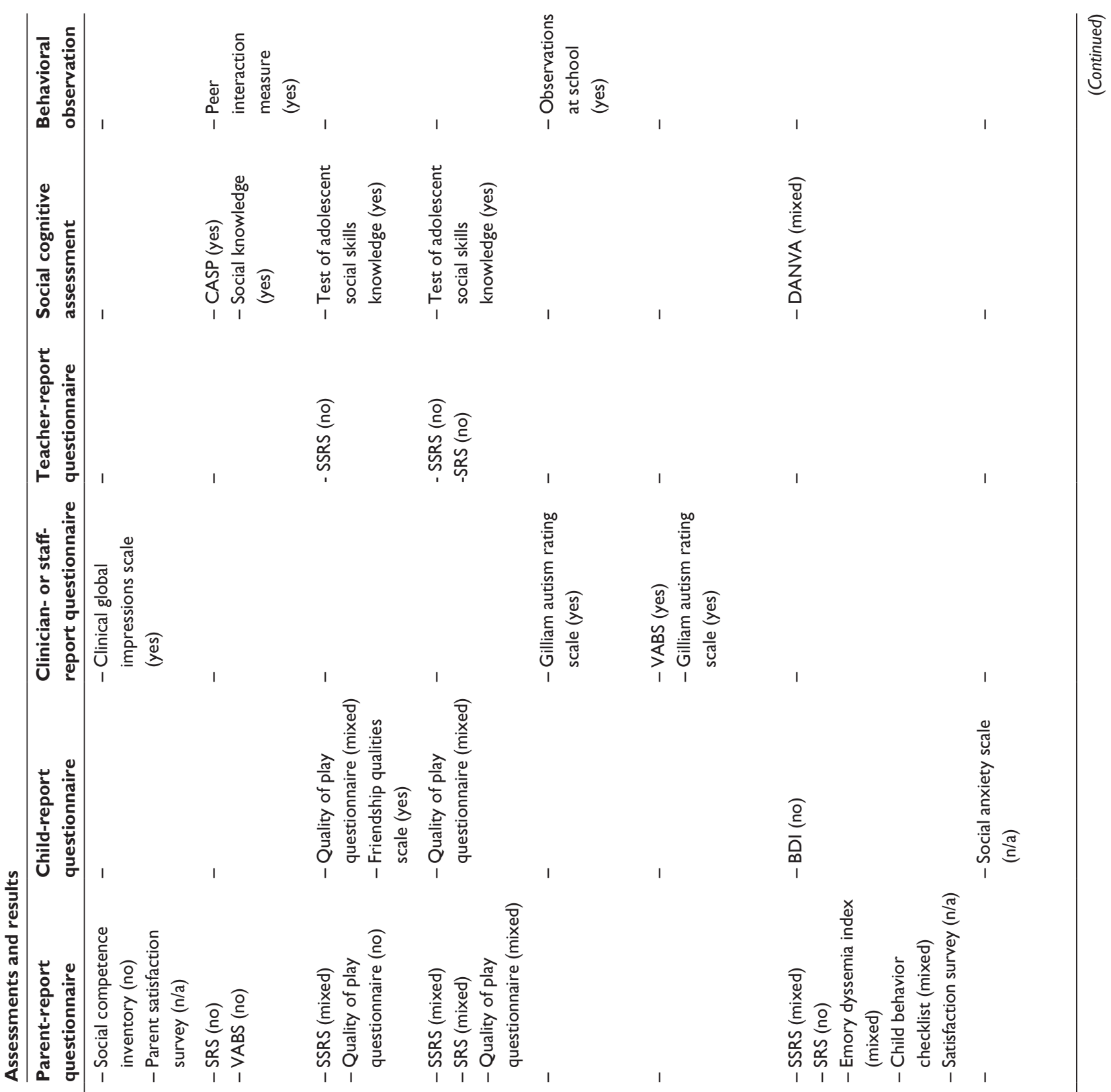

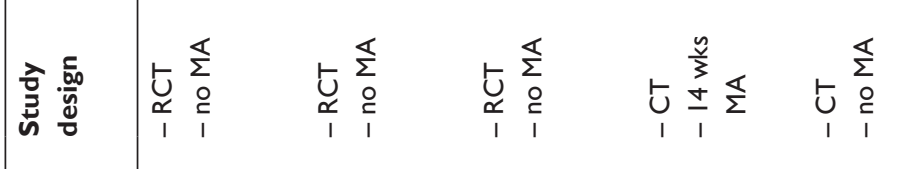
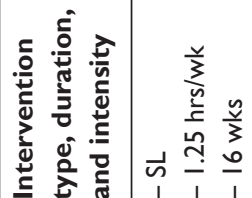

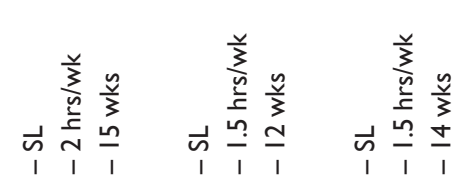

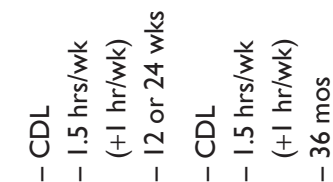

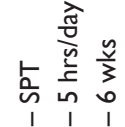

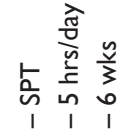

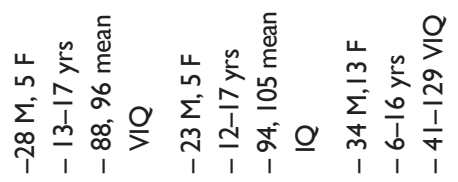

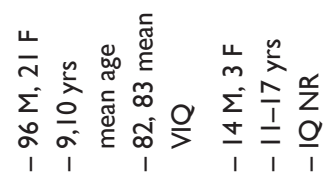

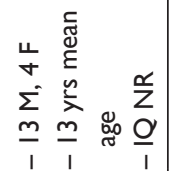

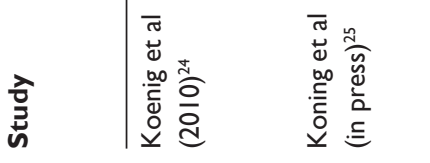

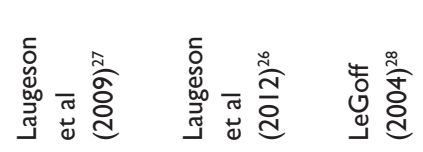

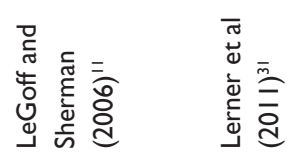

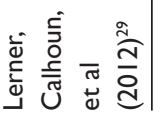




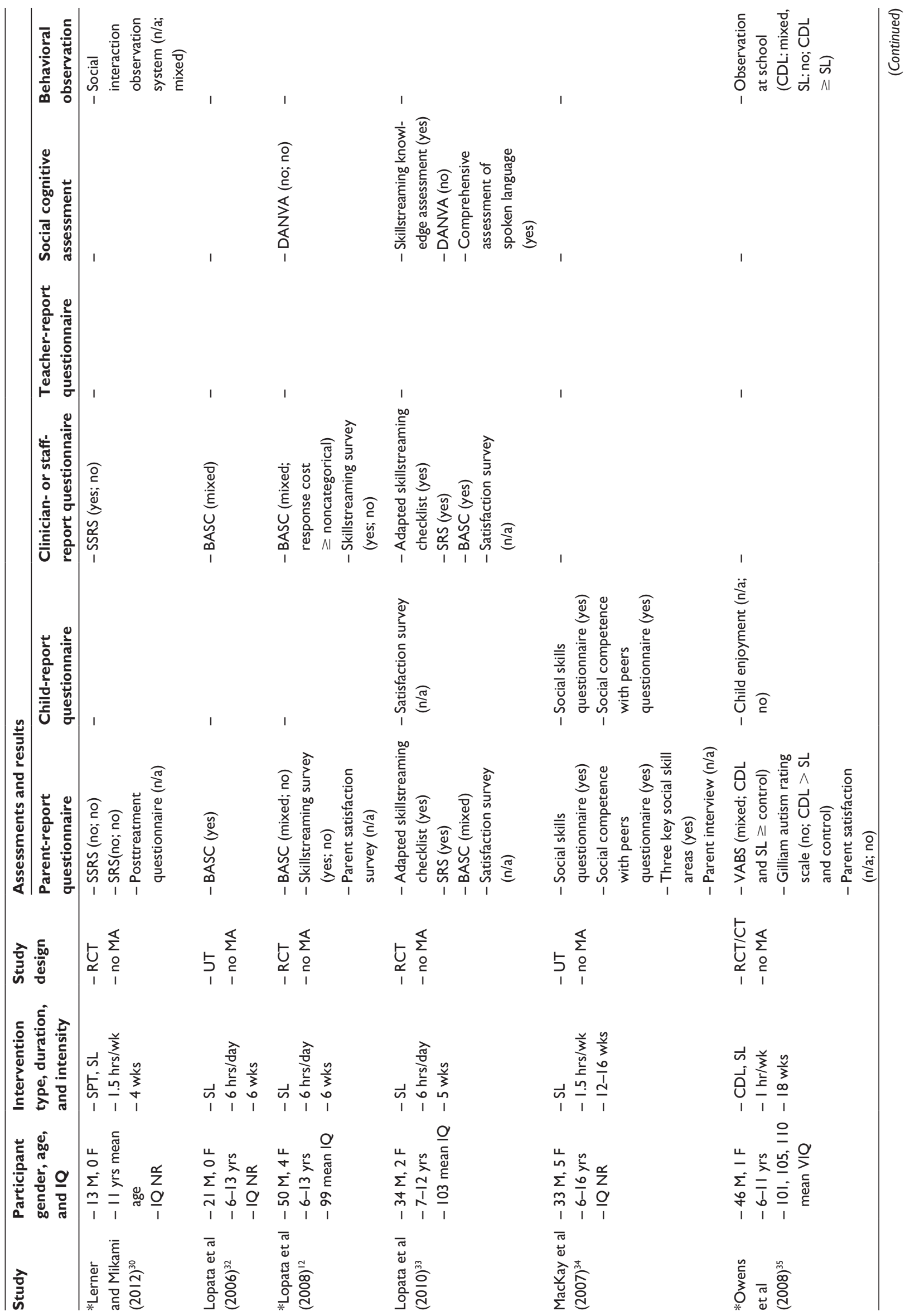



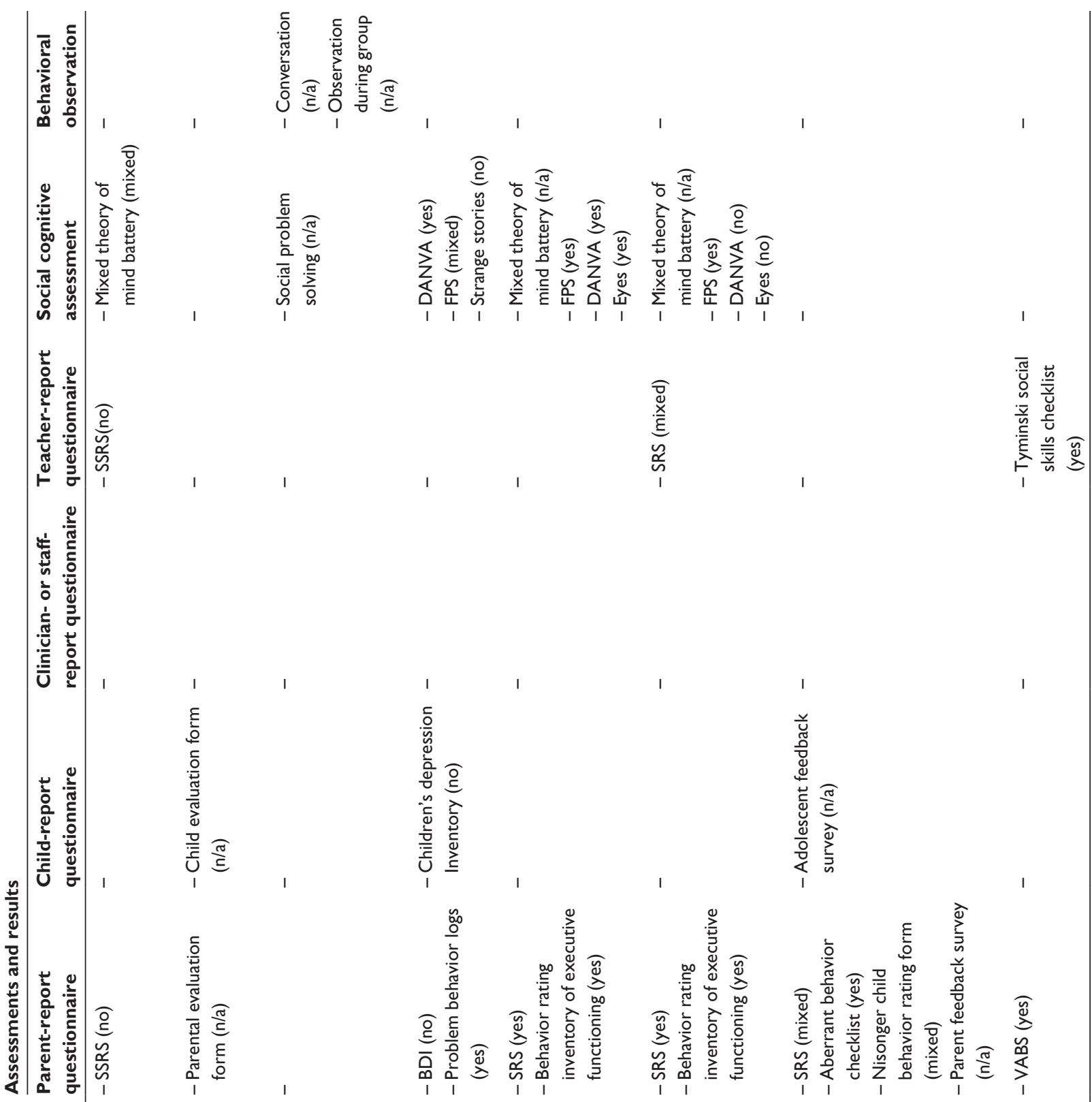

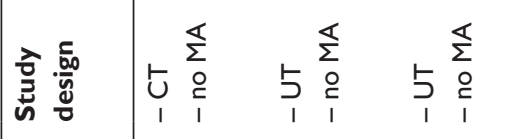

\begin{tabular}{llll}
5 & $\sum_{0}^{\mathbb{E}}$ & & $\sum_{0}^{\mathbb{2}}$ \\
\hdashline & 0 & 5 & 0 \\
1 & 1 & 1 & 1
\end{tabular}

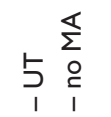

$\underset{\substack{\mathbb{5} \\ 5}}{\substack{0 \\ 1}}$

$\underset{\substack{5 \\ 1}}{\substack{\mathbb{5} \\ 1}}$

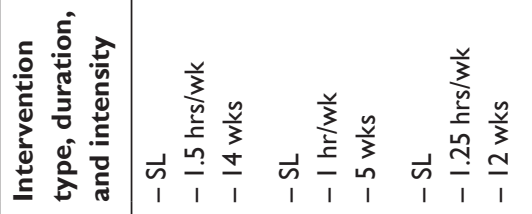

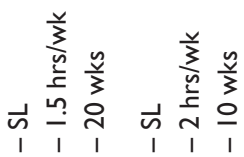

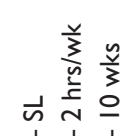

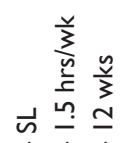

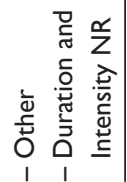

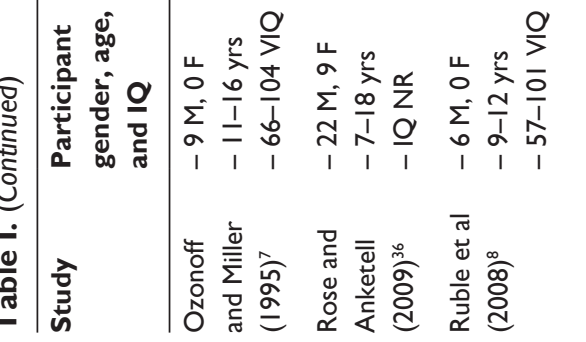

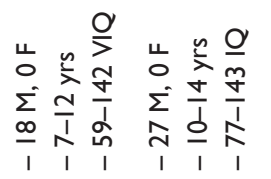

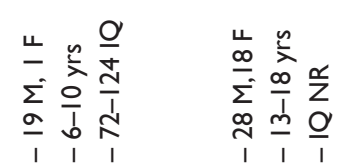

$\begin{array}{ccc}u & 0 & \\ 0 & \frac{2}{\lambda} & \frac{\alpha}{z} \\ \sum_{0} & 0 & 0 \\ m & 1 & 0 \\ 1 & 1 & 1\end{array}$

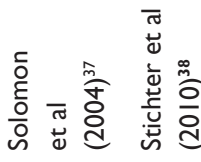

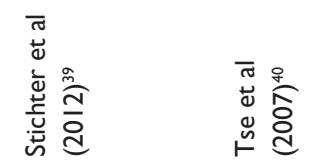

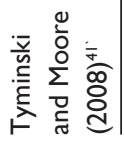




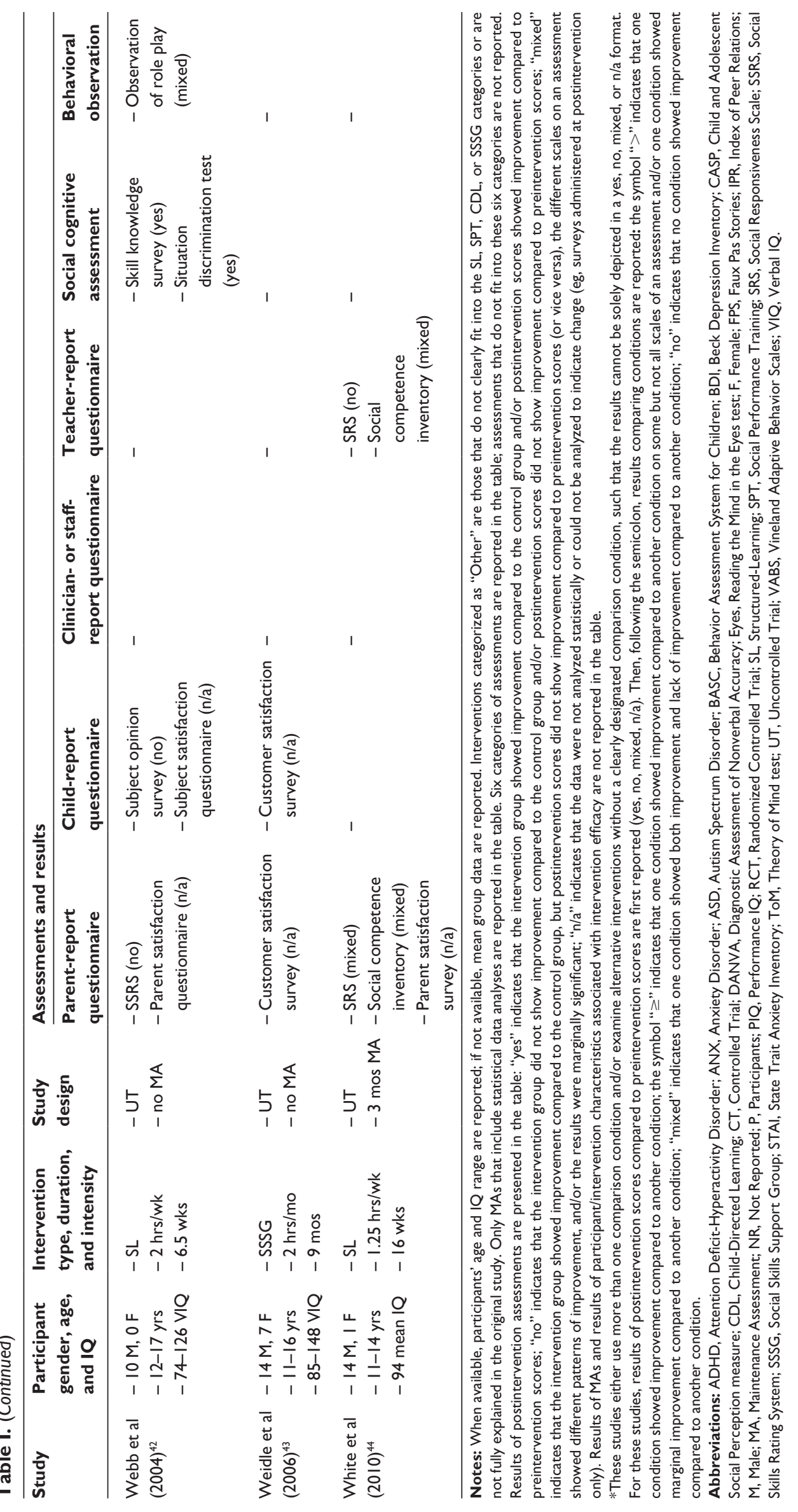


and various questionnaires designed by intervention staff that are specific to the intervention curriculum. ${ }^{17,26,27}$

The most frequently used social cognitive assessment in the GSSI literature is the Diagnostic Assessment of Nonverbal Accuracy. ${ }^{5,12,31,33,37-39,53}$ Other assessments that are occasionally used include the Child and Adolescent Social Perception Measure, ${ }^{15,25,54}$ Reading the Mind in the Eyes test $^{38,39,55}$ Faux Pas Stories, ${ }^{37-39,56}$ Theory of Mind test ${ }^{13,18,57}$ or a mixed theory of mind battery, ${ }^{7,38,39}$ and various assessments designed by intervention staff that are specific to the intervention curriculum. ${ }^{17,26,27,33}$

When behavioral observation is used as an assessment method, participants' behavior is generally observed within the context of a dyadic or group peer interaction. Often, the frequency or duration of participants' interactions, ${ }^{20,23,28,35}$ the appropriateness of the interactions, ${ }^{8,20,23,25}$ and the degree to which participants initiate and/or respond to interactions $\mathbf{s}^{8,23,28,35}$ are coded.

\section{Methods and results}

A randomized controlled trial is the gold-standard method for evaluating the efficacy of a GSSI. ${ }^{58}$ Approximately one-quarter of the studies in the current GSSI literature employ a control group and randomly assign participants

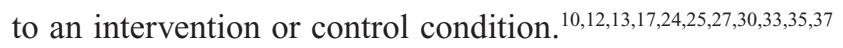
Of these, only a handful of studies ${ }^{10,12,30,35}$ employ a bona fide control group in which control participants receive a viable alternative intervention. ${ }^{59} \mathrm{~A}$ control group enables the researcher to tease apart the effects of the intervention and temporal phenomena (eg, developmental maturation), and a bona fide control group further enables the researcher to tease apart the effects of the actual intervention curriculum and other confounding intervention characteristics (eg, time spent with peers in a supported environment).

There are two methods for identifying efficacious interventions in the GSSI literature. Most commonly, interventions are considered to be efficacious if participants' social skills are significantly improved after the intervention compared to before the intervention. For those interventions that employ a control group, interventions are considered to be efficacious if intervention participants' social skills are significantly improved after the intervention compared to control participants' social skills. Although these methods seem straightforward, "social skills" is a complex construct, ${ }^{60}$ and studies within the GSSI literature tend to report mixed findings, such that significant improvements are seen on some assessments of social skills but not others. In addition, few outcomes are consistently reported across studies within the GSSI literature. Such mixed results may be due to a combination of factors, including participant and intervention characteristics that impact efficacy, inadequate measurement of the social skills construct, and variable study methodology. Given the mixed results within individual GSSI studies and the mixed results across the GSSI literature, few firm conclusions can be drawn about the efficacy of GSSIs.

Of primary interest, it is not clear whether certain GSSI teaching strategies are more efficacious than other teaching strategies. The majority of studies in the GSSI literature assess the efficacy of the structured-learning teaching strategy, while only a few studies assess the efficacy of other teaching strategies, including social performance training, child-directed learning, and social skills support group. The social skills support group strategy, in particular, is in need of further assessment. Thus, the GSSI literature shows a clear disparity in the amount of research that has been done on and our knowledge of these teaching strategies, but the literature does not clearly demonstrate that certain teaching strategies are efficacious or more efficacious than other strategies. Only a few outcomes are consistently reported in the GSSI literature, and even these outcomes have multiple interpretations, as noted below.

Although parent-report and child-report questionnaires tend to yield mixed results, parents and children consistently report high personal satisfaction with GSSIs. ${ }^{5,69,12,14,15,20,22-24,33-}$ ${ }^{36,40,42-44}$ This outcome has received little attention in the GSSI literature, and the interpretation of this outcome is unclear. Parent and child satisfaction may be a meaningful outcome variable; regardless of whether social skills improve as a result of the intervention, parents and children may be better equipped to manage an ASD diagnosis. In addition, satisfaction may indicate the degree to which an intervention is liked or "tolerated" by intervention participants. Alternatively, parent and child satisfaction may be a meaningful mediator of intervention efficacy, such that high satisfaction with the intervention is associated with more social skill improvement. Finally, parent and child satisfaction may be a less meaningful variable that is largely indicative of allegiance to the intervention, investment in the intervention, and/or hope for intervention-related improvements. Satisfaction may also be one of several "common factors" emerging from the process of having individuals with ASD interact in structured settings and may not be indicative of specific intervention characteristics; that is, GSSIs may elicit satisfaction among adolescents with ASD, regardless of the training activities taking place. Parent and child satisfaction is often assessed via informal questionnaires and not subject to statistical 
analyses; more rigorous assessment of this construct is necessary for a clearer interpretation of this outcome.

Teacher-report questionnaires rarely show significant improvements in social skills, even when other assessments indicate such improvements. ${ }^{7,15,19,26,27,44}$ Again, the interpretation of this outcome is unclear. It may be that social skill improvements do not generalize to the classroom. Conversely, social skill improvements may not be appropriately reflected in the classroom; peers may be negatively biased towards individuals with social skill difficulties, even when such individuals improve in their social skills. ${ }^{61}$ As such, teachers may have particular difficulty recognizing and reporting on improvements in social skills. Finally, teachers may not have the opportunity to observe certain social skills in the classroom, thus they may not report improvements in these skills.

Clinician- or intervention staff-report questionnaires frequently show significant improvements in social skills, ${ }^{11,12,19,24,28,30,33}$ although on occasion these questionnaires also indicate increased atypical social behavior. ${ }^{12,32}$ Preintervention assessments are generally completed by clinicians and staff shortly before the intervention or within the first few days of the intervention. It may be that these preintervention assessments do not reliably index participants' behavior: clinicians/staff may not have had adequate opportunities to observe participants' behavior, and participants may be inhibited in their behavior as they acclimate to the intervention setting. ${ }^{12}$ Thus, preintervention assessments may underestimate (or occasionally overestimate) participants' social skills. In addition, clinicians/staff are rarely blind to intervention status, potentially causing postintervention assessments to be positively biased. Finally, participants' gains in social skills may be specific to the intervention setting and may not generalize to home, school, and other settings, such that clinician/staff assessments may be more likely to show improvement in social skills than other assessments. ${ }^{32}$

Participants regularly show improvements on social cognitive assessments designed by intervention staff to index knowledge of social skills targeted in the intervention curriculum. ${ }^{17,26,27,33}$ However, caution should be taken in interpreting these results, as these findings may reflect rote memorization of the social skills curriculum or fidelity of instructors to the administered intervention, rather than a genuine improvement in social skills. As such, it is difficult to know the degree to which these assessments index true intervention efficacy.

Maintenance of social skills is only evaluated by a handful of studies in the GSSI literature, ${ }^{9,15,26,31,44}$ most often at 3 months postintervention, ${ }^{15,44}$ and results regarding maintenance of social skills are mixed. Approximately one-quarter of studies in the GSSI literature evaluate generalization of social skills beyond the intervention setting, either via teacher-report ${ }^{7,15,16,19,26,27,39,41,44}$ or observation of participants' behavior at school. ${ }^{28,35}$ Given that teachers rarely report significant improvements in social skills, there is limited evidence to suggest that social skills generalize beyond the intervention setting. However, as noted earlier, methodological confounds with teacher-report data limit conclusions on generalization at this time.

\section{Group-based social skills interventions: looking to the future Participant characteristics associated with intervention efficacy}

The current GSSI literature has only begun to examine participant characteristics that may mediate (account for some/ all of the relation between the treatment and outcome) or moderate (change the relation between the treatment and outcome) intervention efficacy. ${ }^{62}$ Although no consistent results have emerged, attention deficit-hyperactivity disorder symptomatology,,${ }^{9,13}$ internalizing problems, ${ }^{9}$ ASD severity, ${ }^{7,11,13,24,37,41}$ age, ${ }^{7,19,28,37,41}$ IQ, ${ }^{7,11,19,28,37,41} \operatorname{sex},{ }^{28,41}$ language/communication impairment, ${ }^{11,19,28}$ medication status, ${ }^{19}$ adaptive behavior, ${ }^{34}$ discrepancy between child and parent reports of social skills as an index of child social selfawareness ${ }^{29}$ social cognitive ability ${ }^{41}$ and parent satisfaction with the intervention ${ }^{9}$ have been investigated as potential mediators/moderators of intervention efficacy. The participant's motivation to participate in the intervention, satisfaction with the intervention, sensory responsiveness patterns (eg, hypersensitive, hyposensitive, sensation-seeking), bond with the therapist/teacher and sense of working towards the same goals (ie, therapeutic alliance), and degree of cohesion with members of the social skills group are other potential mediators/moderators that should be considered in future research. ${ }^{62-64}$

\section{Intervention characteristics associated with intervention efficacy}

Recently, a few studies within the GSSI literature have begun to identify and evaluate intervention characteristics that may be associated with intervention efficacy. Sibling involvement in the intervention, ${ }^{15}$ parent involvement in the intervention, ${ }^{10}$ type of performance feedback given to intervention participants, ${ }^{12}$ duration/intensity of the intervention, ${ }^{41}$ and social skills teaching strategy $y^{11,30,35}$ are among the intervention 
characteristics that have been examined. Although the results of such studies require replication, they suggest that future research should continue to evaluate intervention characteristics that may impact efficacy, including the social skills targeted by the intervention, the teaching strategies employed in the intervention, the duration and intensity of the intervention, the setting of the intervention, and the degree to which the intervention adheres to a manual or curriculum.

Pivotal social skills are skills with a widespread impact on social development; an improvement in a pivotal social skill may lead to a cascade of other social skill improvements. ${ }^{65}$ Although pivotal social skills are often identified and discussed in the early intervention literature $\left(\mathrm{eg}\right.$, joint attention $\left.{ }^{66}\right)$, pivotal social skills are rarely identified or discussed in the GSSI literature. Pivotal social skills may play an important role in GSSIs, such that interventions that focus on teaching these skills are more efficacious than other interventions.

Some teaching strategies in the GSSI literature may be more effective than others. ${ }^{11,30,35}$ In particular, it is not clear whether didactic instruction (eg, structured-learning) or implicit instruction (eg, social performance training, childdirected learning) is a more effective method for teaching social skills. Furthermore, there may be "active ingredients" of the social skills curriculum that are particularly essential for learning, maintaining, and/or generalizing social skills. ${ }^{10,12,15}$ Parent involvement, for example, may be critical for generalizing social skills to the home environment, whereas homework assignments may be helpful but not necessary for generalization.

While most interventions either have a shorter session duration and span many weeks (eg, those occurring during the academic year) or a longer session duration and span fewer weeks (eg, those occurring during the summer), it is not clear which intervention schedule is the most effective. Future research is needed to determine the ideal schedule of intervention sessions and whether there is a minimum duration or number of intervention sessions necessary to achieve a desired improvement in social skills.

As the majority of published GSSIs are currently conducted in clinical or university settings, additional research is needed to examine the efficacy of GSSIs conducted in school or community settings. Interventions delivered in these settings, such as those mandated by Individualized Education Programs (IEPs), are often able to reach a wider range of participants. School and community settings may be most familiar to intervention participants, and greater familiarity with the intervention setting may facilitate maintenance and generalization of social skills. In addition, future research should evaluate the effect of fidelity on intervention efficacy; ${ }^{67}$ some GSSIs may only be effective when teachers/therapists strictly adhere to the intervention curriculum, while other GSSIs may be relatively robust to curricular deviations.

Finally, while both participant and intervention characteristics are associated with intervention efficacy, interactions between participant and intervention characteristics may also impact intervention efficacy. ${ }^{68}$ For example, one intervention teaching strategy may be more efficacious for individuals with ASD and comorbid anxiety, while another teaching strategy may be more efficacious for individuals with ASD and comorbid attention deficit-hyperactivity disorder. ${ }^{9}$ Likewise, interventions that target certain social skills may be more efficacious for boys, while interventions that target other social skills may be more efficacious for girls. In addition, some therapeutic settings may be more efficacious for individuals with hypersensitivities, while other therapeutic settings may be more efficacious for individuals with hyposensitivities. Such interaction effects are difficult to detect, but as significant participant and intervention characteristics are identified in the literature, the potential for interaction effects should be considered.

\section{Better measurement of intervention efficacy}

"Social skills" is a complex, multidimensional construct that requires a multimethod measurement approach. ${ }^{60} \mathrm{~A}$ biased, insensitive, or overly simplistic measurement approach can cause effective interventions to be misidentified as ineffective; likewise, ineffective interventions can be incorrectly labeled as effective. In future research, it will be important to recognize and guard against the limitations of common measurement approaches and to develop and use more accurate, sensitive, and comprehensive measurement approaches.

While questionnaires are the most common assessment in the GSSI literature, questionnaires are subjective and informant reports are often not highly correlated. ${ }^{29,69}$ Parent-report and clinician- or intervention staff-report questionnaires are subject to allegiance effects and bias, especially if respondents are not blind to treatment status. Questionnaires completed by clinicians or intervention staff before the intervention may be at increased risk for measurement error if clinicians/ staff are not yet familiar with participants and/or participants are inhibited in their behavior. ${ }^{12}$ Questionnaires should be used in conjunction with more objective assessments, such as social cognitive assessments or behavioral observations. Although it can be logistically difficult, questionnaire 
respondents should be kept blind to treatment status in order to reduce bias and differentiate between intervention and placebo effects. ${ }^{30}$

During evaluation of the efficacy of GSSIs, it is important to consider gains in both social knowledge (ie, knowing a social skill cognitively) and social performance (ie, applying that social skill appropriately). ${ }^{70}$ Gains in social knowledge and performance may not occur concurrently, and both participant characteristics (eg, comorbid attention deficithyperactivity disorder) and intervention characteristics (eg, teaching strategies) may affect gains in these constructs. Social cognitive assessments occur in a structured, lab-based setting; they provide a valuable index of social knowledge, but not necessarily social performance. Thus, social cognitive assessments should be used in conjunction with other measures that evaluate social performance, such as questionnaires or behavioral observations.

While behavioral observation is often time-consuming and resource-intensive, this is the only assessment method that can directly evaluate social skills in a natural environment. Thus, this assessment method has the benefit of ecological validity. ${ }^{71}$ When using this assessment method, behavioral coders should be kept blind to treatment status, receive proper training, and achieve adequate interrater reliability on their coding.

Many assessments that are commonly used in the GSSI literature, such as the Social Responsiveness Scale, ${ }^{45}$ were not designed to evaluate the efficacy of GSSIs, and may therefore have significant limitations in indexing change in social skills over time. In future research, it will be important to develop and use assessments that are directly designed to evaluate interventions and measure temporal changes in social skills.

The GSSI literature should also explore new or different assessment approaches that may more fully capture the multidimensionality of the social skills construct. ${ }^{60}$ Peer-report and sociometric data, although regularly used in other types of literature, ${ }^{72,73}$ have rarely been used in the GSSI literature. ${ }^{30}$ These assessment approaches are often considered gold-standard indicators of peer acceptance and friendship; they provide a valuable peer perspective on social competence that complements data collected from parent, child, teacher, clinician, and/or intervention staff informants. While not yet used in the GSSI literature, neuroimaging and electrophysiological data provide important information on brain-based changes related to intervention, ${ }^{74}$ and a measurement approach that indexes both behavioral and physiological changes may improve understanding of the multidimensionality of the social skills construct.

\section{More rigorous standards for intervention efficacy}

Future studies should use more rigorous methodology for examining the efficacy of GSSIs, and several researchers have identified specific methods that should be employed and criteria that can be used to evaluate the methodological rigor of such studies. ${ }^{75,76}$ Larger sample sizes, inclusion of a bona fide control condition, ${ }^{59}$ random assignment to control and intervention conditions, and assessment of fidelity to the intervention manual are among the key methods that promote rigor and allow researchers to identify efficacious and nonefficacious interventions accurately.

A bona fide control condition is particularly important for determining intervention characteristics that are associated with efficacy. In the current GSSI literature, for example, it is difficult to tease apart the effects of the social skills intervention curriculum and time spent with peers in a supported environment. Peer interaction in a supported environment may lead to social skill improvements even in the absence of an explicit social skills curriculum. ${ }^{22}$ Future research should employ bona fide control conditions to tease apart the effects of the actual intervention curriculum and other subsequent intervention characteristics, such as repeated, supported interactions with peers.

Future researchers should also be more cautious in analyzing data. Often, multiple assessments, with multiple scales on each assessment, are used to determine efficacy in the GSSI literature. While this approach may be intended to capture the multidimensional nature of the social skills construct, ${ }^{60}$ multiple comparisons increase the probability of a Type I error. Future research should employ appropriate statistical controls to guard against chance significance levels. In addition, "statistically significant" should be differentiated from "clinically meaningful." " Statistical significance indicates a genuine difference between two scores; it does not indicate the size or importance of that difference. As such, a small increase in social skills may reach statistical significance, but may not translate into meaningful social skill improvement in daily life. Effect sizes index the magnitude of a statistically significant effect and can be used to provide additional information regarding the clinical utility of such an effect. Lastly, missing data should be considered when evaluating intervention efficacy. ${ }^{78}$ If data are not missing at random (eg, intervention participants with fewer social skill improvements were more likely to drop out of the intervention), this confound should be addressed in the analyses. 
While the current literature predominantly focuses on the positive effects of GSSIs, future research should also consider potential negative effects of GSSIs. ${ }^{79}$ Increased social selfawareness, for example, may allow intervention participants to recognize and address social skill impairments more effectively; however, greater awareness of such impairments may lead to symptoms of depression and anxiety. ${ }^{29,37,80}$ Future research should be more attentive to these potential negative side effects, such that efficacy is determined by evaluating both positive and potential negative effects.

Finally, future research should more rigorously and routinely assess for maintenance and generalization of social skills. Such research should consider which social skills (eg, social knowledge, social performance ${ }^{70}$ ) are maintained over time and the maintenance trajectories of those skills. Certain social skills may be more readily maintained over time, which would have important implications for the design and implementation of GSSIs. Future research should also carefully evaluate generalization of social skills to the classroom setting; in the current literature, it is not clear whether social skills do not generalize to the classroom setting and/or whether such generalization is obscured by social-contextual factors in the classroom. ${ }^{61}$ Lastly, social skill improvements that are short-lived and/or restricted to the intervention setting are not clinically meaningful improvements. As such, assessments of maintenance and generalization of social skills should occur regularly in the GSSI literature and should be considered essential for establishing the efficacy of an intervention.

\section{Conclusion}

In this paper, we have considered the research participants, the intervention, the assessment of the intervention, and the research methodology and results to be integral and interconnected components of the GSSI literature. Given the current research, no specific GSSI can be said to be uniformly efficacious for individuals with ASD. However, emerging research provides several promising directions. In the future, we hope that more sophisticated and rigorous research methodology will lend clarity to key questions regarding intervention efficacy.

\section{Acknowledgments}

Preparation of this manuscript was partially supported by a T32 Institutional Research Training Grant from the National Institute of Mental Health (PI: Sally Rogers, 5-T32MH073124-06) to Camilla M McMahon, as well as fellowships from the American Psychological Foundation and Jefferson Scholars Foundation to Matthew D Lerner.

\section{Disclosure}

The authors have no conflicts of interest to disclose.

\section{References}

1. American Psychiatric Association. Diagnostic and Statistical Manual of Mental Disorders. 4th ed. Washington, DC: American Psychiatric Association; 2000.

2. Autism and Developmental Disabilities Monitoring Network Surveillance Year 2008 Principal Investigators; Centers for Disease Control and Prevention. Prevalence of autism spectrum disorders - Autism and Developmental Disabilities Monitoring Network, 14 sites, United States, 2008. MMWR Surveill Summ. 2012;61(3):1-19.

3. Interagency Autism Coordinating Committee. The 2011 Interagency Autism Coordinating Committee Strategic Plan for Autism Spectrum Disorder Research. 2011. Available from: http://iacc.hhs.gov/strategicplan/2011/index.shtml. Accessed July 28, 2012.

4. Lerner M, Hileman C, Britton N. Promoting the social and emotional development of adolescents with autism spectrum disorder. In: Gullotta T, Leukefeld C, editors. Encyclopedia of Primary Prevention and Health Promotion: Adolescence. 2nd ed. New York: Springer; 2012. In press.

5. Barnhill GP, Tapscott Cook K, Tebbenkamp K, Smith Myles B. The effectiveness of social skills intervention targeting nonverbal communication for adolescents with Asperger syndrome and related pervasive developmental delays. Focus Autism Other Dev Disabl. 2002;17(2):112-118

6. Broderick C, Caswell R, Gregory S, Marzolini S, Wilson O. 'Can I join the club?': a social integration scheme for adolescents with Asperger syndrome. Autism. 2002;6(4):427-431.

7. Ozonoff S, Miller JN. Teaching theory of mind: a new approach to social skills training for individuals with autism. J Autism Dev Disord. 1995;25(4):415-433.

8. Ruble L, Willis H, McLaughlin Crabtree V. Social skills group therapy for autism spectrum disorders. Clin Case Stud. 2008;7(4):287-300.

9. Antshel KM, Polacek C, McMahon M, et al. Comorbid ADHD and anxiety affect social skills group intervention treatment efficacy in children with autism spectrum disorders. J Dev Behav Pediatr. 2011;32(6): 439-446.

10. DeRosier ME, Swick DC, Davis NO, McMillen JS, Matthews R. The efficacy of a social skills group intervention for improving social behaviors in children with high functioning autism spectrum disorders. J Autism Dev Disord. 2011;41(8):1033-1043.

11. Legoff DB, Sherman M. Long-term outcome of social skills intervention based on interactive LEGO play. Autism. 2006;10(4):317-329.

12. Lopata C, Thomeer ML, Volker MA, Nida RE, Lee GK. Effectiveness of a manualized summer social treatment program for high-functioning children with autism spectrum disorders. J Autism Dev Disord. 2008;38(5):890-904.

13. Begeer S, Gevers C, Clifford P, et al. Theory of Mind training in children with autism: a randomized controlled trial. J Autism Dev Disord. 2011;41(8):997-1006.

14. Carter C, Meckes L, Pritchard L, Swensen S, Wittman PP, Velde B. The Friendship Club: an after-school program for children with Asperger syndrome. Fam Community Health. 2004;27(2):143-150.

15. Castorina LL, Negri LM. The inclusion of siblings in social skills training groups for boys with Asperger syndrome. J Autism Dev Disord. 2011;41(1):73-81.

16. Cotugno AJ. Social competence and social skills training and intervention for children with autism spectrum disorders. JAutism Dev Disord. 2009;39(9):1268-1277.

17. Gantman A, Kapp SK, Orenski K, Laugeson EA. Social skills training for young adults with high-functioning autism spectrum disorders: a randomized controlled pilot study. J Autism Dev Disord. 2012;42(6): 1094-1103.

18. Gevers C, Clifford P, Mager M, Boer F. Brief report: a theory-of-mindbased social-cognition training program for school-aged children with pervasive developmental disorders: an open study of its effectiveness. J Autism Dev Disord. 2006;36(4):567-571. 
19. Herbrecht E, Poustka F, Birnkammer S, et al. Pilot evaluation of the Frankfurt Social Skills Training for children and adolescents with autism spectrum disorder. Eur Child Adolesc Psychiatry. 2009;18(6): $327-335$.

20. Hillier A, Fish T, Cloppert P, Beversdorf DQ. Outcomes of a social and vocational skills support group for adolescents and young adults on the autism spectrum. Focus Autism Other Dev Disabl. 2007;22(2): 107-115.

21. Hillier AJ, Fish T, Siegel JH, Beversdorf DQ. Social and vocational skills training reduces self-reported anxiety and depression among young adults on the autism spectrum. J Dev Phys Disabil. 2011;23(3): 267-276.

22. Hillier A, Greher G, Poto N, Dougherty M. Positive outcomes following participation in a music intervention for adolescents and young adults on the autism spectrum. Psychol Music. 2012;40(2): 201-215.

23. Howlin P, Yates P. The potential effectiveness of social skills groups for adults with autism. Autism. 1999;3(3):299-307.

24. Koenig K, White SW, Pachler M, et al. Promoting social skill development in children with pervasive developmental disorders: a feasibility and efficacy study. J Autism Dev Disord. 2010;40(10):1209-1218.

25. Koning C, Magill-Evans J, Volden J, Dick B. Efficacy of cognitive behavior therapy-based social skills intervention for school-aged boys with autism spectrum disorders. Res Autism Spectr Disord. 2012. In press.

26. Laugeson EA, Frankel F, Gantman A, Dillon AR, Mogil C. Evidencebased social skills training for adolescents with autism spectrum disorders: the UCLA PEERS program. J Autism Dev Disord. 2012; 42(6):1025-1036.

27. Laugeson EA, Frankel F, Mogil C, Dillon AR. Parent-assisted social skills training to improve friendships in teens with autism spectrum disorders. J Autism Dev Disord. 2009;39(4):596-606.

28. LeGoff DB. Use of LEGO as a therapeutic medium for improving social competence. J Autism Dev Disord. 2004;34(5):557-571.

29. Lerner MD, Calhoun CD, Mikami AY, De Los Reyes A. Understanding parent-child social informant discrepancy in youth with high functioning autism spectrum disorders. J Autism Dev Disord. 2012;42:2680-2692.

30. Lerner MD, Mikami AY. A preliminary randomized controlled trial of two social skills interventions for youth with high-functioning autism spectrum disorders. Focus Autism Other Dev Disabl. 2012;27(3): 147-157.

31. Lerner MD, Mikami AY, Levine K. Socio-dramatic affective-relational intervention for adolescents with Asperger syndrome and high functioning autism: pilot study. Autism. 2011;15(1):21-42.

32. Lopata C, Thomeer ML, Volker MA, Nida RE. Effectiveness of a cognitive-behavioral treatment on the social behaviors of children with Asperger disorder. Focus Autism Other Dev Disabl. 2006;21(4): 237-244.

33. Lopata C, Thomeer ML, Volker MA, et al. RCT of a manualized social treatment for high-functioning autism spectrum disorders. J Autism Dev Disord. 2010;40(11):1297-1310.

34. Mackay T, Knott F, Dunlop AW. Developing social interaction and understanding in individuals with autism spectrum disorder: a groupwork intervention. J Intellect Dev Disabil. 2007;32(4):279-290.

35. Owens G, Granader Y, Humphrey A, Baron-Cohen S. LEGO therapy and the social use of language programme: an evaluation of two social skills interventions for children with high functioning autism and Asperger syndrome. J Autism Dev Disord. 2008;38(10): 1944-1957.

36. Rose R, Anketell C. The benefits of social skills groups for young people with autism spectrum disorder: a pilot study. Child Care Pract. 2009;15(2):127-144.

37. Solomon M, Goodlin-Jones BL, Anders TF. A social adjustment enhancement intervention for high functioning autism, Asperger's syndrome, and pervasive developmental disorder NOS. J Autism Dev Disord. 2004;34(6):649-668.

38. Stichter JP, Herzog MJ, Visovsky K, et al. Social competence intervention for youth with Asperger syndrome and high-functioning autism: an initial investigation. J Autism Dev Disord. 2010;40(9): 1067-1079.
39. Stichter JP, O'Connor KV, Herzog MJ, Lierheimer K, McGhee SD. Social competence intervention for elementary students with Aspergers syndrome and high functioning autism. J Autism Dev Disord. 2012; 42(3):354-366.

40. Tse J, Strulovitch J, Tagalakis V, Meng L, Fombonne E. Social skills training for adolescents with Asperger syndrome and high-functioning autism. J Autism Dev Disord. 2007;37(10):1960-1968.

41. Tyminski RF, Moore PJ. The impact of group psychotherapy on social development in children with pervasive developmental disorders. Int $J$ Group Psychother. 2008;58(3):363-379.

42. Webb BJ, Miller SP, Pierce TB, Strawser S, Jones WP. Effects of social skill instruction for high-functioning adolescents with autism spectrum disorders. Focus Autism Other Dev Disabl. 2004;19(1): 53-62.

43. Weidle B, Bolme B, Hoeyland, AL. Are peer support groups for adolescents with Asperger's syndrome helpful? Clin Child Psychol Psychiatry. 2006;11(1):45-62.

44. White SW, Koenig K, Scahill L. Group social skills instruction for adolescents with high-functioning autism spectrum disorders. Focus Autism Other Dev Disabl. 2010;25(4):209-219.

45. Constantino J, Gruber C. Social Responsiveness Scale. Los Angeles: Western Psychological Services; 2005.

46. Gresham F, Elliott S. The Social Skills Rating System. Circle Pines $(\mathrm{MN})$ : American Guidance Service; 1990.

47. Reynolds C, Kamphaus R. Behavior Assessment System for Children. 2nd ed. Circle Pines (MN): American Guidance Service; 2004.

48. Sparrow S, Cicchetti D, Balla D. Vineland Adaptive Behavior Scales. 2nd ed. Minneapolis: NCS Pearson; 2005.

49. Beck J, Beck A, Jolly J. Beck Youth Inventories. San Antonio: Psychological Corporation; 2001.

50. Beck A, Steer R, Brown G. Beck Depression Inventory. San Antonio: Psychological Corporation; 1996.

51. Hudson W. The Index of Peer Relations. Tallahassee (FL): Walmyr Publishing Company; 1992.

52. Spielberger C, Gorsuch R, Lushene P, Vagg P, Jacobs A. Manual for the State-Trait Anxiety Inventory (Form Y). Palo Alto (CA): Mind Garden; 1983.

53. Nowicki S. Instructional Manual for the Receptive Tests of the Diagnostic Analysis of Nonverbal Accuracy 2. Atlanta: Peachtree;1997.

54. Magill-Evans J, Koning C, Cameron-Sadava A, Manyk K. The child and adolescent social perception measure. J Nonverbal Behav. 1995;19(3):151-169.

55. Baron-Cohen S, Wheelwright S, Hill J, Raste Y, Plumb I. The "reading the mind in the eyes" test revised version: a study with normal adults, and adults with Asperger syndrome or high-functioning autism. J Child Psychol Psychiatry. 2001;42(2):241-251.

56. Baron-Cohen S, O'Riordan M, Stone V, Jones R, Plaisted K. Recognition of faux pas by normally developing children with Asperger syndrome or high-functioning autism. J Autism Dev Disord. 1999;29(5): $407-418$.

57. Muris P, Steerneman P, Meesters C, et al. The TOM test: a new instrument for assessing theory of mind in normal children and children with pervasive developmental disorders. J Autism Dev Disord. 1999;29(1): 67-80.

58. Smith T, Scahill L, Dawson G, et al. Designing research studies on psychosocial interventions in autism. J Autism Dev Disord. 2007;37(2):354-366.

59. Wampold BE, Mondin GW, Moody M, Stich F, Benson K, Ahn H. A meta-analysis of outcome studies comparing bona fide psychotherapies: empiricially, "all must have prizes." Psychol Bull. 1997;122(3): 203-215.

60. Koenig K, De Los Reyes A, Cicchetti D, Scahill L, Klin A. Group intervention to promote social skills in school-age children with pervasive developmental disorders: reconsidering efficacy. J Autism Dev Disord. 2009;39(8):1163-1172.

61. Mikami AY, Lerner MD, Lun J. Social context influences on children's rejection by their peers. Child Dev Perspect. 2010;4(2):123-130. 
62. Lerner MD, White SW, McPartland JC. Mechanisms of change in psychosocial interventions for autism spectrum disorders. Dialogues Clin Neurosci. 2012;14:307-318.

63. Briesch AM, Chafouleas SM. Exploring student buy-in: initial development of an instrument to measure likelihood of children's intervention usage. J Educ Psychol Consult. 2009;19(4):321-336.

64. Norcross JC, Wampold BE. Evidence-based therapy relationships: research conclusions and clinical practices. Psychotherapy (Chic). 2011;48(1):98-102.

65. Koegel RL, Frea WD. Treatment of social behavior in autism through the modification of pivotal social skills. J Appl Behav Anal. 1993; 26(3):369-377.

66. Charman T. Why is joint attention a pivotal skill in autism? Philos Trans $R$ Soc Lond B Biol Sci. 2003;358(1430):315-324.

67. Dane A, Schneider BH. Program integrity in primary and early secondary prevention: Are implementation effects out of control? Clin Psychol Rev. 1998;18(1):23-45.

68. Smith B, Sechrest L. Treatment of aptitude $\times$ treatment interactions. J Consult Clin Psychol. 1991;59(2):233-244.

69. De Los Reyes A, Kazdin AE. Informant discrepancies in the assessment of childhood psychopathology: a critical review, theoretical framework, and recommendations for further study. Psychol Bull. 2005;131(4): 483-509.

70. Gresham FM. Social competence and students with behavior disorders: where we've been, where we are, and where we should go. Educ Treat Children. 1997;20(3):233-249.

71. Elliott SN, Gresham FM. Children's social skills: assessment and classification practices. J Couns Dev. 1987;66(2):96-99.
72. Hoza B, Mrug S, Gerdes AC, et al. What aspects of peer relationships are impaired in children with attention-deficit/hyperactivity disorder? J Consult Clin Psychol. 2005;73(3):411-423.

73. Kasari C, Rotheram-Fuller E, Locke J, Gulsrud A. Making the connection: randomized controlled trial of social skills at school for children with autism spectrum disorders. J Child Psychol Psychiatry. 2012;53(4):431-439.

74. Faja S, Webb SJ, Jones E, et al. The effects of face expertise training on the behavioral performance and brain activity of adults with high functioning autism spectrum disorders. J Autism Dev Disord. 2012;42(2): 278-293.

75. Nathan P, Gorman J, editors. A Guide to Treatments That Work. 3rd ed. New York: Oxford University Press; 2007.

76. Reichow B, Volkmar FR, Cicchetti DV. Development of the evaluative method for evaluating and determining evidence-based practices in autism. J Autism Dev Disord. 2008;38(7):1311-1319.

77. Jacobson NS, Truax P. Clinical significance: a statistical approach to defining meaningful change in psychotherapy research. $J$ Consult Clin Psychol. 1991;59(1):12-19.

78. Gould MS, Shaffer D, Kaplan D. The characteristics of dropouts from a child psychiatry clinic. J Am Acad Child Psychiatry. 1985;24(3): 316-328.

79. Lilienfeld SO. Psychological treatments that cause harm. Perspect Psychol Sci. 2007;2(1):53-70.

80. Verhoeven EW, Marijnissen N, Berger HJ, Oudshoorn J, van der Sijde A, Teunisse JP. Brief report: relationship between self-awareness of realworld behavior and treatment outcome in autism spectrum disorders. J Autism Dev Disord. 2012;42(5):889-894.
Adolescent Health, Medicine and Therapeutics

\section{Publish your work in this journal}

Adolescent Health, Medicine and Therapeutics is an international, peer-reviewed, open access journal focusing on health, pathology, and treatment issues specific to the adolescent age group. All aspects of health maintenance, preventative measures and disease treatment interventions are addressed within the journal and practitioners from

\section{Dovepress}

all disciplines are invited to submit their work as well as healthcare researchers and patient support groups.. The manuscript management system is completely online and includes a very quick and fair peerreview system. Visit http://www.dovepress.com/testimonials.php to read real quotes from published authors. 